\title{
A comparison between different semiclassical approximations for optical response functions in nonpolar liquid solutions
}

\author{
Qiang Shi and Eitan Geva \\ Department of Chemistry, University of Michigan, Ann Arbor, Michigan 48109-1055
}

(Received 6 August 2004; accepted 10 November 2004; published online 1 February 2005)

\begin{abstract}
The temporal behavior of optical response functions (ORFs) reflects the quantum dynamics of an electronic superposition state, and as such lacks a well-defined classical limit. In this paper, we consider the importance of accounting for the quantum nature of the dynamics when calculating ORFs of different types. To this end, we calculated the ORFs associated with the linear absorption spectrum and the nonlinear two-pulse photon-echo experiment, via the following approaches: (1) the semiclassical forward-backward approach; (2) an approach based on linearizing the path-integral forward-backward action in terms of the difference between the forward and backward paths; (3) an approach based on ground state nuclear dynamics. The calculations were performed on a model that consists of a two-state chromophore solvated in a nonpolar liquid. The different methods were found to yield very similar results for the absorption spectrum and "diagonal" two-pulse photon echo (i.e., the homodyne-detected signal at time $t=t_{0}$ after the second pulse, where $t_{0}$ is the time interval between the two pulses). The different approximations yielded somewhat different results in the case of the time-integrated photon-echo signal. The reasons for the similarity between the predictions of different approximations are also discussed (c) 2005 American Institute of Physics.
\end{abstract}

[DOI: $10.1063 / 1.1843813]$

\section{INTRODUCTION}

Many experimental and theoretical studies over the last two decades have been targeted at elucidating the structure and dynamics of liquid solutions. The development of ultrafast lasers, in particular, has given rise to many new nonlinear time-domain techniques which are capable of directly probing solvation dynamics on the femtosecond time scale. ${ }^{1-6}$ The measured spectroscopic signals are often expressed by optical response function (ORFs), which can be given in terms of material multitime dipole correlation functions. ${ }^{3}$ The temporal behavior of the ORFs reflects the time evolution of the system in an electronic superposition state, which lacks a well-defined classical limit. Unfortunately, with the exception of a few simplified models, the exact calculation of the corresponding quantum-mechanical dipole correlation functions remains far beyond the reach of currently available computer resources.

Several approximate methods for calculating ORFs in anharmonic many-body systems, such as liquid solutions, have been suggested in the past. ${ }^{3,7-16}$ Most of those methods are based on treating the nuclear degrees of freedom (DOF) in a classical-like manner. However, a straightforward implementation of a classical treatment for those DOF leads to a nonunique procedure, due to the lack of a well-defined classical limit. More specifically, the form of the actual classical limit that one ends up with depends on the form of the expression for the quantum mechanical ORF that one chooses to start with. ${ }^{8,9,11-14,17,18}$ For example, a commonly used approach is based on using one particular form of the quantummechanical ORF, whose "classical limit" happens to dictate that the nuclear dynamics is performed on the potential surface that corresponds to the ground electronic state. The tem- poral behavior of ORFs computed via this approximation reflects equilibrium fluctuations in the nuclear dynamics on the ground electronic state surface. Taking the classical limit of other equivalent forms of the quantum-mechanical ORF leads to different prescriptions. Indeed, a variety of alternative propagation schemes have been discussed in the literature, including exclusive propagation on the excited surface, ${ }^{9,11,13,14}$ propagation that alternates between the ground and excited surfaces, ${ }^{3,17}$ and propagation on an average surface. ${ }^{8,12,17}$ However, it is not clear which, if any, of these strategies will work best for a given system.

In a series of recent papers, we have explored the use of an approximate scheme which is based on linearizing the path-integral forward-backward action with respect to the difference between the forward and backward paths. ${ }^{19-24} \mathrm{We}$, as well as other researchers, have found that the resulting approximations are computationally feasible, and yield rather accurate results, in a variety of contexts, including vibrational energy relaxation, ${ }^{20-22}$ nonradiative electronic relaxation ${ }^{18,25}$ the calculation of reaction rate constants, ${ }^{26}$ ORFs, ${ }^{8,12}$ and nonadiabatic dynamics. ${ }^{23,24}$

An alternative approach can be based on the semiclassical initial value representation (SC-IVR) method, ${ }^{27-35}$ which has also been found to be rather reliable in a variety of applications, including the calculation of spectroscopic observables. ${ }^{33-42}$ A calculation based on the full blown implementation of the SC-IVR approximation is not feasible in the case of condensed phase systems, mainly due to the highly oscillatory nature of the integrand. Several approaches have been proposed in order to make the calculation more manageable, including the semiclassical cellular dynamics method of Mukamel and co-workers, ${ }^{3,36}$ the 
mixed-state propagation approximation proposed by Loring and co-workers, ${ }^{38,39}$ the mixed-order semiclassical approach proposed by Ovchinnikov and co-workers, ${ }^{34,40,43,44}$ and methods based on Filinov and generalized Filinov filters. ${ }^{33,35,41,42}$ An alternative, and rather different, approach has been recently proposed by Makri and co-workers. ${ }^{45-49}$ In this so-called forward-backward initial value representation (FB-IVR) method, the forward and backward propagators, as well as the operator between them, are treated as a single propagator. This makes the calculation more manageable, because the partial cancellation of the forward and backward paths usually results in mildly oscillatory integrands and tames the rapid increase in the prefactor of the semiclassical propagator. This approach is particularly suitable for calculating ORFs within the framework of the Condon approximation, where the dipole operator is assumed to be independent of the nuclear DOF, and where the dipole correlation function only involves time propagators. Recent applications that demonstrate this include the calculation of the photoelectron spectrum of $\mathrm{I}_{2}^{-}$in the gas phase by Batista and co-workers, ${ }^{49}$ the calculation of the absorption spectrum for $\mathrm{I}_{2}$ molecule interacting with a chain of argon atoms by Kühn and Makri, ${ }^{15}$ and the calculation of the resonance Raman spectrum of an $\mathrm{I}_{2}$ molecule in liquid xenon by Ovchinnikov and co-workers. ${ }^{16}$

The primary goal of the present paper is to explore the importance of accounting for the quantum nature of the dynamics when calculating ORFs of different types. To this end, we present a systematic comparison between the FB-IVR approximation and simpler semiclassical approximations, in the case of the linear and nonlinear two-pulse photon-echo (2PE) ORFs in a nonpolar liquid solution. We hope that such an analysis will shed light on the sensitivity of different experimentally relevant quantities to quantum dynamical effects. The remainder of this paper is organized in the following way: A survey of the theory, the various approximations, and the assumptions underlying them, is given in Sec. II. The results obtained via the various approximations for the absorption spectrum and 2PE signal, in the case of a two-state chromophore solvated in a nonpolar liquid solution, are reported and discussed in Sec. III. The main conclusions are summarized in Sec. IV.

\section{THEORY}

\section{A. Optical response functions}

We consider a system with two electronic states, whose overall Hamiltonian is given by

$$
\hat{H}=\hat{H}_{g}|g\rangle\left\langle g\left|+\hat{H}_{e}\right| e\right\rangle\langle e|-E(t)\left(\mu_{e g}|e\rangle\left\langle g\left|+\mu_{g e}\right| g\right\rangle\langle e|\right) .
$$

Here, $g$ and $e$ correspond to the ground and excited electronic states, $E(t)$ is the (classical) driving electromagnetic field, and $\left\{\mu_{i j}\right\}$ are the transition dipole moments (assumed to be constant within the Condon approximation). The nuclear Hamiltonians $\hat{H}_{g}$ and $\hat{H}_{e}$ are assumed to have the following general form:

$$
\hat{H}_{\alpha}(\hat{\mathbf{p}}, \hat{\mathbf{q}})=\sum_{j=1}^{N} \frac{\hat{p}_{j}^{2}}{2 m_{j}}+V_{\alpha}(\hat{\mathbf{q}}),
$$

where $\quad \alpha=g, \quad e, \quad \hat{\mathbf{q}}=\left(\hat{q}_{1}, \ldots, \hat{q}_{N}\right), \quad \hat{\mathbf{p}}=\left(\hat{p}_{1}, \ldots, \hat{p}_{N}\right), \quad$ and $\left(m_{1}, \ldots, m_{N}\right)$ are the coordinates, momenta, and masses of the corresponding nuclear DOF (boldface and capped symbols for vectors and operators, respectively, are used throughout the text). We also assume that $\hbar \omega_{e g} / k_{B} T \gg 1$, where $\hbar \omega_{e g}$ is the energy gap between the bath-free electronic states, $T$ is the absolute temperature, and $k_{B}$ is the Boltzmann constant.

As is well known, the absorption line shape is given by the Fourier transform of the quantum-mechanical dipole autocorrelation function, which for the model described above is given by ${ }^{3,13,14}$

$$
\mathrm{I}(\omega)=\frac{1}{2 \pi} \int_{-\infty}^{\infty} d t e^{-i \omega t} J(t),
$$

where

$$
J(t)=Z_{g}^{-1} \operatorname{Tr}\left\{e^{-\beta \hat{H}_{g}} e^{i \hat{H}_{e} t / \hbar} e^{-i \hat{H}_{g} t / \hbar}\right\} .
$$

Here, $\beta=1 / k_{B} T, \operatorname{Tr}[\cdots]$ corresponds to tracing over the nuclear DOF and $Z_{g}=\operatorname{Tr}\left[e^{-\beta \hat{H}_{g}}\right]$ is the partition function at the ground electronic state. The function $J(t)$ is the sought after linear ORF, which stores the desired information on solute-solvent interactions.

Similarly, the signal in the homodyne-detected 2PE experiment is known to be given by ${ }^{3,9}$

$$
\mathrm{I}_{2 \mathrm{PE}}\left(t, t_{0}\right)=\left|R\left(t, t_{0}\right)\right|^{2},
$$

where $R\left(t, t_{0}\right)$ is the corresponding nonlinear ORF:

$R\left(t, t_{0}\right)=Z_{g}^{-1} \operatorname{Tr}\left\{e^{-\beta \hat{H}_{g}} e^{(i / \hbar) \hat{H}_{e} t_{0}} e^{(i / \hbar) \hat{H}_{g} t} e^{-(i / \hbar) \hat{H}_{e} t} e^{-(i / \hbar) \hat{H}_{g} t_{0}}\right\}$.

Here, $t_{0}$ and $t$ are the time intervals between the two pulses, and between the second pulse and the time of the measurement, respectively. It should be noted that $2 \mathrm{PE}$ signals measured in liquid solutions are typically "smeared" over an extended time interval, rather than given by a sharp echo at $t$ $=t_{0}$ (as in crystalline and glassy hosts). The results of $2 \mathrm{PE}$ experiments are therefore often reported in terms of the integrated echo, which is given by

$$
\mathrm{I}_{2 \mathrm{PE}}^{i n t}\left(t_{0}\right)=\int_{0}^{\infty} d t\left|R\left(t, t_{0}\right)\right|^{2} .
$$

\section{B. Semiclassical approximations for the optical response functions}

A numerically exact calculation of the quantummechanical ORFs in Eqs. (4) and (6) is not possible in the general case of many-body anharmonic systems, such as liquid solutions. In this section, we review three different approximate schemes which give rise to more feasible routes for calculating those ORFs.

A widely used computational scheme for computing ORFs is based on the so called dynamical classical limit (DCL) approximation, ${ }^{18,25}$ which leads to the following ap- 
proximate expressions for the linear and $2 \mathrm{PE}$ response functions:

$$
\begin{aligned}
J_{\mathrm{DCL}}(t)= & \left(Z_{g}^{C l}\right)^{-1} \iint d \mathbf{q} d \mathbf{p} e^{-\beta H_{g}(\mathbf{q}, \mathbf{p})} \\
& \times \exp \left[\frac{i}{\hbar} \int_{0}^{t} d t^{\prime} U_{g}\left(t^{\prime}\right)\right]
\end{aligned}
$$

and

$$
\begin{aligned}
R_{\mathrm{DCL}}\left(t, t_{0}\right)= & \left(Z_{g}^{C l}\right)^{-1} \iint d \mathbf{q} d \mathbf{p} e^{-\beta H_{g}(\mathbf{q}, \mathbf{p})} \\
& \times \exp \left[\frac{i}{\hbar} \int_{0}^{\left|t_{0}\right|} d t^{\prime} U_{g}\left(t^{\prime}\right)-\frac{i}{\hbar} \operatorname{sgn}\left(t_{0}\right)\right. \\
& \left.\times \int_{\left|t_{0}\right|}^{\left|t_{0}\right|+t} d t^{\prime} U_{g}\left(t^{\prime}\right)\right] .
\end{aligned}
$$

The sampling of the initial state in Eqs. (8) and (9) is based on the classical Boltzmann distribution $e^{-\beta H_{g}(\mathbf{q}, \mathbf{p})} / Z_{g}^{C L}$, which corresponds to the ground state potential surface. Importantly, $U_{g}(t)=V_{e}\left[\mathbf{q}_{g}(t)\right]-V_{g}\left[\mathbf{q}_{g}(t)\right]$ corresponds to the vertical energy gap between the two electronic states, and its time evolution is dictated by that of $\mathbf{q}_{g}(t)$, which is governed by classical dynamics on the ground state potential.

$J_{\mathrm{DCL}}(t)$ and $R_{\mathrm{DCL}}\left(t, t_{0}\right)$ can be computed from equilibrium classical molecular dynamics (MD) simulations on the ground state potential surface. At the same time, Eqs. (8) and (9) do not correspond to a unique classical limit of Eqs. (4) and (6), as was previously pointed out by several authors. $3,8,9,11,12,18,38,39,50$ An argument that is often made in favor of the DCL approximation is that the absorption line shape of a chromophore in liquid solution is usually inhomogeneously broadened, and is therefore insensitive to the underlying dynamics. ${ }^{9,13,14}$ More specifically, if the lifetime of the linear ORF is shorter than the characteristic time scale of the nuclear DOF, then $J_{\mathrm{DCL}}(t)$ can be replaced by its inhomogeneous limit:

$J_{i n h}(t)=\left(Z_{g}^{C l}\right)^{-1} \iint d \mathbf{q} d \mathbf{p} e^{-\beta H_{g}(\mathbf{q}, \mathbf{p})} \exp \left[\frac{i}{\hbar} U(\mathbf{q}) t\right]$,

which is indeed insensitive to the dynamics. However, this argument is not valid in the case of nonlinear optical experiments, such as photon echoes, which are specifically designed to expose dynamical effects by eliminating inhomogeneous broadening. In those cases, one may argue that, at least in the limit where $V_{g}$ and $V_{e}$ are not too different, it does not matter which surface the system is propagated on. ${ }^{9}$ However, to the best of our knowledge, no systematic analysis of the validity of this argument has been performed. In fact, the popularity of of the DCL approximation in actual calculations of ORFs in liquid solutions is probably best explained by its relative simplicity, as well as by its extensive use in the earlier literature on spectroscopy in lowtemperature hosts. ${ }^{51-55}$

The second computational scheme to be considered has been proposed in the past for calculating the absorption line shape, ${ }^{18,25}$ nonradiative electronic relaxation rate constants, ${ }^{56}$ and the photon-echo signal. ${ }^{8,12}$ It is based on the Wigner averaged classical limit (WACL) approximation. ${ }^{25}$ One way of deriving this approximation is by linearizing the forwardbackward action in the corresponding path integral expressions for the ORF, with respect to the difference between the forward and backward paths. ${ }^{24}$ The WACL approximations for the linear and 2PE ORFs are given by

$$
\begin{aligned}
J_{\mathrm{WACL}}(t)= & \frac{1}{(2 \pi \hbar)^{N}} \int d \mathbf{q}_{0} d \mathbf{p}_{0} \rho_{w}\left(\mathbf{q}_{0}, \mathbf{p}_{0}\right) \\
& \times \exp \left\{i \int_{0}^{t} d t^{\prime} U_{a v}\left(t^{\prime}\right) / \hbar\right\}
\end{aligned}
$$

and

$$
\begin{aligned}
R_{\mathrm{WACL}}\left(t, t_{0}\right)= & \iint d \mathbf{q} d \mathbf{p} \rho_{w}(\mathbf{q}, \mathbf{p}) \exp \left[\frac{i}{\hbar} \int_{0}^{\left|t_{0}\right|} d t^{\prime} U_{a v}\left(t^{\prime}\right)\right. \\
& \left.-\frac{i}{\hbar} \operatorname{sgn}\left(t_{0}\right) \int_{\left|t_{0}\right|}^{\left|t_{0}\right|+t} d t^{\prime} U_{a v}\left(t^{\prime}\right)\right]
\end{aligned}
$$

respectively. Here, $\rho_{w}(\mathbf{q}, \mathbf{p})$ is the Wigner-Weyl transform of the initial density operator

$$
\begin{aligned}
\rho_{w}(\mathbf{q}, \mathbf{p})= & Z_{g}^{-1} \int d \boldsymbol{\Delta} \exp \{-i \mathbf{p} \cdot \boldsymbol{\Delta} / \hbar\} \\
& \times\left\langle\mathbf{q}+\boldsymbol{\Delta} / 2\left|e^{-\beta \hat{H}_{g}}\right| \mathbf{q}-\mathbf{\Delta} / 2\right\rangle,
\end{aligned}
$$

with $\Delta=\left(\Delta_{1}, \ldots, \Delta_{N}\right)$, and the temporal behavior of $U_{a v}\left(t^{\prime}\right)$ is dictated by classical dynamics on the averaged potential surface:

$$
V_{a v}(\mathbf{q})=\frac{1}{2}\left[V_{g}(\mathbf{q})+V_{e}(\mathbf{q})\right] .
$$

The third computational scheme to be considered is based on the semiclassical FB-IVR approximation, recently proposed by Makri co-workers. ${ }^{15,45-49}$ The ORFs in Eqs. (4) and (6) are particularly suitable for this approximation, since they only involve time-evolution operators, which is the result of employing the Condon approximation. Within the FB-IVR approximation, one treats the product of timeevolution operators in Eqs. (4) and (6) as a single timeevolution operator, and approximate it by the corresponding semiclassical Herman-Kluk propagator. ${ }^{30,31}$ The FB-IVR approximations for the linear and $2 \mathrm{PE}$ ORFs are given by ${ }^{15,16}$

$$
\begin{aligned}
J_{\mathrm{FB}}(t)= & \frac{Z_{g}^{1}}{(2 \pi \hbar)^{N}} \int d \mathbf{q}_{0} d \mathbf{p}_{0} D\left(\mathbf{p}_{0}, \mathbf{q}_{0}\right) \\
& \times\left\langle\mathbf{p}_{0}, \mathbf{q}_{0}\left|e^{-\beta \hat{H}_{g}}\right| \mathbf{p}_{f}, \mathbf{q}_{f}\right\rangle e^{i S_{a b s}\left(\mathbf{p}_{0}, \mathbf{q}_{0}\right) / \hbar}
\end{aligned}
$$

and

$$
\begin{aligned}
R_{\mathrm{FB}}\left(t, t_{0}\right)= & \frac{Z_{g}^{1}}{(2 \pi \hbar)^{N}} \int d \mathbf{q}_{0} d \mathbf{p}_{0} D\left(\mathbf{p}_{0}, \mathbf{q}_{0}\right) \\
& \times\left\langle\mathbf{p}_{0}, \mathbf{q}_{0}\left|e^{-\beta \hat{H}_{g}}\right| \mathbf{p}_{f}, \mathbf{q}_{f}\right\rangle e^{i / \hbar S_{2 \mathrm{PE}}\left(\mathbf{p}_{0}, \mathbf{q}_{0}\right)},
\end{aligned}
$$

respectively. Here, $|\mathbf{p}, \mathbf{q}\rangle$ corresponds to a coherent state of the form 


$$
\begin{aligned}
\langle\mathbf{x} \mid \mathbf{p}, \mathbf{q}\rangle= & \left(\frac{1}{\pi}\right)^{N / 4}(\operatorname{det} \gamma)^{1 / 4} \exp \left[-\frac{1}{2}(\mathbf{x}-\mathbf{q})^{T} \gamma(\mathbf{x}-\mathbf{q})\right. \\
& \left.+\frac{i}{\hbar} \mathbf{p} \cdot(\mathbf{x}-\mathbf{q})\right],
\end{aligned}
$$

with $\gamma=\gamma \mathbf{I}$, where $\mathbf{I}$ is the identity matrix and $\gamma$ is a positive constant. ${ }^{16}$ The corresponding FB actions are given by

$$
\begin{aligned}
S_{a b s}\left(\mathbf{q}_{0}, \mathbf{p}_{0}\right)= & \int_{0}^{t}\left[\mathbf{p} \cdot \dot{\mathbf{q}}-H_{g}(\mathbf{p}, \mathbf{q})\right] d \tau \\
& +\int_{t}^{0}\left[\mathbf{p} \cdot \dot{\mathbf{q}}-H_{e}(\mathbf{p}, \mathbf{q})\right] d \tau
\end{aligned}
$$

and

$$
\begin{aligned}
S_{2 \mathrm{PE}}\left(\mathbf{q}_{0}, \mathbf{p}_{0}\right)= & \int_{0}^{t_{0}}\left[\mathbf{p} \cdot \dot{\mathbf{q}}-H_{g}(\mathbf{p}, \mathbf{q})\right] d \tau+\int_{t_{0}}^{t_{0}+t}[\mathbf{p} \cdot \dot{\mathbf{q}} \\
& \left.-H_{e}(\mathbf{p}, \mathbf{q})\right] d \tau+\int_{t_{0}+t}^{t_{0}}\left[\mathbf{p} \cdot \dot{\mathbf{q}}-H_{g}(\mathbf{p}, \mathbf{q})\right] d \tau \\
& +\int_{t_{0}}^{0}\left[\mathbf{p} \cdot \dot{\mathbf{q}}-H_{e}(\mathbf{p}, \mathbf{q})\right] d \tau
\end{aligned}
$$

$\mathbf{q}_{f}$ and $\mathbf{p}_{f}$ are the coordinates and momenta at the end of the corresponding forward-backward propagations, and $D\left(\mathbf{p}_{0}, \mathbf{q}_{0}\right)$ is the familiar Herman-Kluk semiclassical prefactor. $^{30,31}$

The statistical weights of trajectories in the WACL and FB-IVR approximations are dictated by $\rho_{w}\left(\mathbf{q}_{0}, \mathbf{p}_{0}\right)$ and $\left\langle\mathbf{p}_{0}, \mathbf{q}_{0}\left|e^{-\beta H_{g}}\right| \mathbf{p}_{f}, \mathbf{q}_{f}\right\rangle / Z_{g}$, respectively. In practice, it is necessary to resort to additional approximations in order to compute those wights in the case of a liquid solution. One possibility is based on replacing $\rho_{w}\left(\mathbf{q}_{0}, \mathbf{p}_{0}\right)$ and $\left\langle\mathbf{p}_{0}, \mathbf{q}_{0}\left|e^{-\beta H_{g}}\right| \mathbf{p}_{f}, \mathbf{q}_{f}\right\rangle / Z_{g}$ by their high-temperature limit. The high-temperature approximation (HTA) of $\rho_{w}\left(\mathbf{q}_{0}, \mathbf{p}_{0}\right)$ reduces to the corresponding classical Boltzmann distribution. The explicit expression for the HTA of $\left\langle\mathbf{p}_{0}, \mathbf{q}_{0}\left|e^{-\beta \hat{H}_{g}}\right| \mathbf{p}_{f}, \mathbf{q}_{f}\right\rangle / Z_{g}$, which lacks a well-defined classical analog, can be found in Refs. 15, 45, and 47.

An alternative approach can be based on a local harmonic approximation (LHA) ${ }^{20,21}$ In the case of WACL, this corresponds to expanding $V_{g}$ in $\left\langle\mathbf{q}+\boldsymbol{\Delta} / 2\left|e^{-\beta \hat{H}_{g}}\right| \mathbf{q}-\boldsymbol{\Delta} / 2\right\rangle$, to second order around $\mathbf{q}$, and explicitly solving the resulting Gaussian integral (cf. Refs. 20 and 21 for a more detailed discussion). The same approximation can be employed in the case of the FB-IVR approximation, by using the following identity:

$$
\begin{aligned}
\left\langle\mathbf{p}_{0}, \mathbf{q}_{0}\left|e^{-\beta \hat{H}_{g}}\right| \mathbf{p}_{f}, \mathbf{q}_{f}\right\rangle= & \int d \mathbf{q} d \boldsymbol{\Delta}\left\langle\mathbf{p}_{0}, \mathbf{q}_{0} \mid \mathbf{q}+\Delta / 2\right\rangle \\
& \times\left\langle\mathbf{q}+\Delta / 2\left|e^{-\beta \hat{H}_{g}}\right| \mathbf{q}-\boldsymbol{\Delta} / 2\right\rangle\langle\mathbf{q} \\
& -\Delta / 2\left|\mathbf{p}_{f}, \mathbf{q}_{f}\right\rangle,
\end{aligned}
$$

followed by a LHA for $\left\langle\mathbf{q}+\boldsymbol{\Delta} / 2\left|e^{-\beta \hat{H}_{g}}\right| \mathbf{q}-\boldsymbol{\Delta} / 2\right\rangle$ and explicitly solving the corresponding Gaussian integrals. The nu- merical implementation of those schemes is similar to that in Refs. 15, 16, 20, and 21.

\section{APPLICATION TO A NONPOLAR LIQUID SOLUTION}

In this section, we present the results obtained for the linear and 2PE response functions via the various approximations surveyed in Sec. II in the case of a two-state chromophore in a nonpolar liquid solution. The goal of this analysis is to estimate the accuracy of the approximations, as well as the sensitivity of different observables to the quantum nature of the underlying dynamics. ${ }^{3,7-9,13,14,57,58}$ The calculations were performed for a model that constituted of a single two-state chromophore in a 2D liquid solution. ${ }^{59}$ Twenty five solvent atoms were included in a 2D simulation cell with periodic boundary conditions, in order to minimize the relatively high cost of computing the FB-IVR approximation. The potential energy surfaces of the ground and excited electronic states are assumed to be pairwise additive, such that

$$
V_{\alpha}=\sum_{k} v_{\alpha}\left(r_{k}\right)+\sum_{k<j} v_{s}\left(r_{k j}\right) .
$$

Here, $r_{k}$ is the distance between the chromophore and the $k$ th solvent atom; and $r_{k j}$ is the distance between the $k$ th and $j$ th solvent atoms. It should be noted that the chromophoresolvent interactions in the excited state are assumed to be different from those in the ground state, whereas the solventsolvent interactions are the same in both states.

The actual pair potentials $v_{g}, v_{e}$, and $v_{s}$ were adopted from Ref. 13, where they were used for analyzing ORFs in nonpolar liquid solutions, within the context of the DCL approximation. More specifically, the pair potentials $v_{g}, v_{e}$, and $v_{s}$ are assumed to have the form of Lennard-Jones (LJ) potentials:

$$
v(r)=4 \epsilon\left[\left(\frac{\sigma}{r}\right)^{12}-\left(\frac{\sigma}{r}\right)^{6}\right],
$$

and we assume that $v_{g}=v_{s}$, with LJ parameters that correspond to liquid argon, namely, $\epsilon / k_{B}=119.8 \mathrm{~K}$ and $\sigma$ $=3.405 \AA . v_{e}$ is assumed to be similar to $v_{g}$, except for the fact that $\sigma_{e}=(1+\lambda) \sigma_{g}$, with $\lambda=0.06$. ${ }^{13}$ We also assume that the chromophore mass is equal to that of the solvent atoms, and corresponds to the mass of an argon atom. MD simulations were carried out at a temperature $T=128.2 \mathrm{~K}$ and density $\rho=0.0604 \AA^{-2}$ (the corresponding reduced LJ temperature and density are given by $T^{*}=k_{B} T / \epsilon_{g}=1.07$ and $\rho^{*}$ $=\rho \sigma_{g}^{2}=0.7$, respectively).

The initial sampling of the coordinates in the case of the LHA-WACL and LHA-FB-IVR approximations was performed via a PIMD simulation. ${ }^{20}$ Each DOF was represented by a six-bead necklace, and the corresponding classical equations of motion were integrated by using the velocity Verlet algorithm. ${ }^{60,61}$ The system was equilibrated over $10^{6}$ time steps, each of length $1.5 \times 10^{-3}$ (in reduced units), by attaching a two-element Nose-Hoover chain to each of the beads. ${ }^{60,61}$ The parameter $\gamma$ was set to $2 m / \beta \hbar^{2}$ in the FB- 


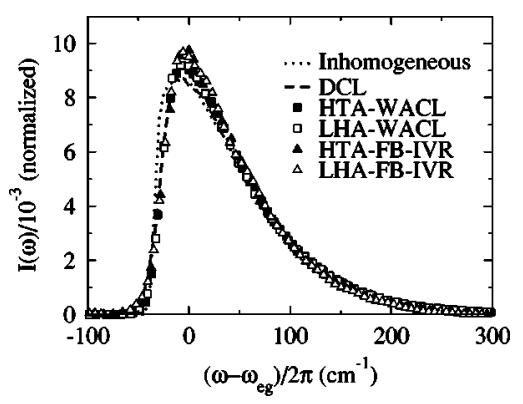

FIG. 1. The absorption spectrum of a two-state chromophore in liquid solution, as obtained via the DCL, HTA-WACL, LHA-WACL, HTA-FB-IVR, and LHA-FB-IVR approximations, as well as at the inhomogeneous limit.

IVR simulations. Converged results for the DCL, WACL, and FB-IVR approximations were obtained by averaging over $10^{4}-10^{5}$ trajectories.

The absorption spectra for this model, as obtained via the DCL, HTA-WACL, LHA-WACL, HTA-FB-IVR, and LHA-FB-IVR approximations, as well as at the inhomogeneous limit, are shown in Fig. 1. The absorption line shape is observed to be dominated by inhomogeneous broadening, which is manifested by the great similarity between the DCL and inhomogeneous spectra. ${ }^{13,14}$ The line shapes predicted by the HTA-WACL, LHA-WACL, HTA-FB-IVR, and LHAFB-IVR approximations are similar, and deviate only slightly in comparison to the prediction of the DCL approximation. Those observations are consistent with the expected insensitivity of the linear absorption spectrum to dynamics, as well as to quantum effects in the initial sampling.

The diagonal homodyne-detected 2PE signal at time $t$ $=t_{0}, \mathrm{I}_{2 \mathrm{PE}}\left(t_{0}, t_{0}\right)$, is given in Fig. 2, as obtained via the DCL, LHA-WACL, and LHA-FB-IVR approximations. The integrated 2PE signal is shown as a function of $t_{0}$ in Fig. 3, as obtained via the DCL, LHA-WACL, and LHA-FB-IVR approximations. The results obtained for the diagonal and integrated 2PE via HTA-WACL and HTA-FB-IVR essentially coincide with the LHA-WACL and LHA-FB-IVR results, respectively, and are therefore not shown explicitly. $\mathrm{I}_{2 \mathrm{PE}}\left(t_{0}, t_{0}\right)$ is seen to be completely insensitive to the type of approximation used in order to describe the dynamics. On the other hand, the DCL approximation of the integrated $2 \mathrm{PE}, \mathrm{I}_{2 \mathrm{PE}}^{\text {int }}\left(t_{0}\right)$, is noticeably different from the WACL and FB-IVR approximations, which give very similar predictions. Thus, from all

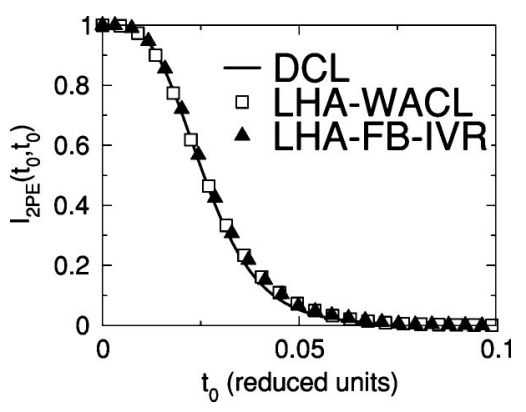

FIG. 2. The homodyne-detected two-photon-echo signal for a two-state chromophore in liquid solution, at $t=t_{0}$. Shown are the DCL, LHA-WACL, and LHA-FB-IVR approximations (the HTA-WACL and HTA-FB-IVR approximations are indistinguishable from the LHA-WACL and LHA-FB-IVR approximations, and are therefore not shown explicitly).

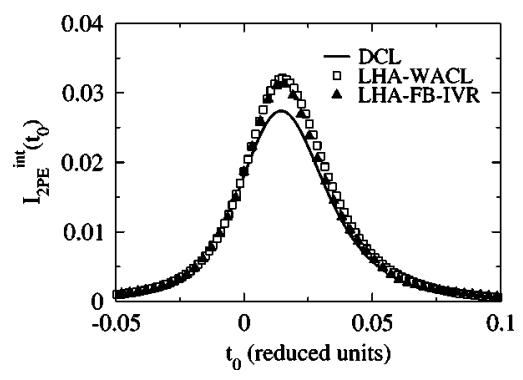

FIG. 3. The integrated two-photon-echo signal for a two-state chromophore in liquid solution, as a function of $t_{0}$. Shown are the exact, DCL, LHAWACL, and LHA-FB-IVR approximations (the HTA-WACL and HTA-FBIVR approximations are indistinguishable from the LHA-WACL and LHAFB-IVR approximations, and are therefore not shown explicitly).

the quantities considered, only the integrated $2 \mathrm{PE}$ signal is observed to be somewhat sensitive to the quantum nature of the underlying dynamics.

The fact that using the HTA and LHA gave essentially identical results for all quantities considered, implies that the nuclear DOF can be treated as classical (as far as the initial sampling is concerned). The observation that the absorption spectrum is insensitive to the quantum nature of the underlying dynamics is due to the fact that, for the model considered, the line shape is inhomogeneously broadened, and reflects initial sampling rather than the subsequent time evolution. It should be noted that this conclusion may not necessarily hold in cases where the ORF is sensitive to bound intramolecular vibrational dynamics. This possibility is suggested by the findings in Ref. 18, where the DCL and WACL approximations of the linear ORF were compared with the exact quantum-mechanical result in the case of a two-state harmonic diatomic molecule bilinearly coupled to a harmonic bath. The authors of Ref. 18 have found that the DCL approximation was unable to capture vibronic structure in the absorption spectrum, and that the WACL approximation was able to capture the vibronic structure in some cases, but failed to do so in other cases. At the same time, the recent work of Ovchinnikov et al., who used the FB-IVR method in order to compute the nonlinear ORF that corresponds to the resonance Raman spectrum of $\mathrm{I}_{2}$ in liquid xenon, seems to suggest that the FB-IVR approximation is able to accurately capture the vibronic structure. ${ }^{16}$

It is important to note the complete insensitivity of the diagonal $2 \mathrm{PE}$ signal, $\mathrm{I}_{2 \mathrm{PE}}\left(t_{0}, t_{0}\right)$, to the quantum nature of the underlying dynamics (cf. Fig. 2). A similar behavior has been previously pointed out by Shemetulskis and Loring, who found that the DCL and WACL approximations gave essentially identical predictions for $\mathrm{I}_{2 \mathrm{PE}}\left(t_{0}, t_{0}\right)$ in the case of a fluid consisting of dipolar soft spheres. ${ }^{8}$ Those authors have attributed this insensitivity to the fact that the decay of $\mathrm{I}_{2 \mathrm{PE}}\left(t_{0}, t_{0}\right)$ reflects inertial motion of solvent molecules, and is therefore insensitive to the dynamics dictated by the potential energy surfaces. The fact that in our case, the prediction of FB-IVR for $\mathrm{I}_{2 \mathrm{PE}}\left(t_{0}, t_{0}\right)$ is essentially indistinguishable from the prediction of the DCL and WACL approximations seem to reinforce the validity of this argument. However, it should be emphasized that our results also show that the off-diagonal component of the 2PE signal, which contributes to the inte- 
grated 2PE signal, is at least somewhat sensitive to the quantum nature of the noninertial dynamics (cf. Fig. 3). Furthermore, the fact that the difference between DCL and WACL/ FB-IVR is not large in our case, is at least partly due to the fact that $V_{e}$ and $V_{g}$ are rather similar in the case of a nonpolar liquid solution. It would obviously be desirable to extend the analysis to polar liquid solutions, where the stronger interactions may lead to more pronounced deviations, and/or to the case of the three-pulse photon echo, which is more sensitive to solvation dynamics on longer time scales. ${ }^{9}$ However, calculating the relevant quantities via the FB-IVR approximation would be much more demanding in those cases, and is therefore reserved to future study.

\section{SUMMARY}

We have performed a comparison between the DCL, WACL, and FB-IVR approximations, in the context of linear and nonlinear ORFs of a two-state solute in a nonpolar liquid solution. In the case of the WACL and FB-IVR approximations, we have also considered different approximations for sampling the initial state, namely HTA and LHA. The results suggest that the linear ORF is insensitive to the quantum nature of both the initial sampling and the dynamics. As a result, all different methods give essentially the same absorption spectrum.

In retrospect, one could have anticipated this result based on the fact that the absorption line shape is dominated by inhomogeneous broadening, and the relatively large mass of the nuclei. At the same time, the nonlinear 2PE ORF was found to be somewhat sensitive to the quantum nature of the dynamics, as long as one looks at the overall, rather than the diagonal, signal. We have also established that the results obtained via the FB-IVR method are very similar to those obtained via the WACL approximation. Thus, the accuracy of those two methods seem to be comparable, at least for the model considered here.

Although the WACL and FB-IVR approximations for the integrated 2PE clearly deviate from the prediction of the DCL approximation, the discrepancy was found to be rather small. This is at least partly due to the fact that the ground and excited potential surfaces in the model considered are rather similar. Larger differences between the two potential surfaces, as in the case of a polar solvent-solute system, would probably give rise to larger discrepancies, and a more pronounced signature of the quantum nature of the underlying dynamics. However, those systems will also be characterized by larger FB actions, such that converging the FB-IVR calculation would become increasingly more demanding. It should also be noted that other quantities, such as the heterodyne-detected $2 \mathrm{PE}$ and ORFs that correspond to other nonlinear optical experiments, such as the three-pulse photon echo, may be more sensitive to the quantum nature of the dynamics. Those issues are currently under investigation in our group, and will be reported in future publications.

\section{ACKNOWLEDGMENT}

The authors are grateful to the National Science Foundation for financial support, through Grant No. CHE0306695 .

${ }^{1}$ P. Vöhringer, D. C. Arnett, T.-S. Yang, and N. F. Scherer, Chem. Phys. Lett. 237, 387 (1995)

${ }^{2}$ R. M. Stratt and M. Maroncelli, J. Phys. Chem. 100, 12981 (1996).

${ }^{3}$ S. Mukamel, Principles of Nonlinear Optical Spectroscopy (Oxford University Press, New York, 1995).

${ }^{4}$ W. P. de Boeij, M. S. Pshenichnikov, and D. A. Wiersma, Annu. Rev. Phys. Chem. 49, 99 (1998).

${ }^{5}$ G. R. Fleming and M. Cho, Annu. Rev. Phys. Chem. 47, 109 (1996).

${ }^{6}$ A. B. Myers, Annu. Rev. Phys. Chem. 49, 267 (1998).

${ }^{7}$ A. M. Walsh and R. F. Loring, Chem. Phys. Lett. 186, 77 (1991).

${ }^{8}$ N. E. Shemetulskis and R. F. Loring, J. Chem. Phys. 97, 1217 (1992).

${ }^{9}$ K. F. Everitt, E. Geva, and J. L. Skinner, J. Chem. Phys. 114, 1326 (2001).

${ }^{10}$ J. L. Skinner, J. Chem. Phys. 77, 3398 (1982).

${ }^{11}$ S. Mukamel, J. Chem. Phys. 77, 173 (1982).

${ }^{12}$ B. D. Bursulaya and H. J. Kim, J. Phys. Chem. 100, 16451 (1996).

${ }^{13}$ M. D. Stephens, J. G. Saven, and J. L. Skinner, J. Chem. Phys. 106, 2129 (1997).

${ }^{14}$ J. G. Saven and J. L. Skinner, J. Chem. Phys. 99, 4391 (1993).

${ }^{15}$ O. Kühn and N. Makri, J. Phys. Chem. A 103, 9487 (1999).

${ }^{16}$ M. Ovchinnikov, V. A. Apkarian, and G. A. Voth, J. Chem. Phys. 184, 7130 (2001).

${ }^{17}$ L. E. Fried, N. B. Bernstein, and S. Mukamel, Phys. Rev. Lett. 68, 1842 (1992).

${ }^{18}$ S. A. Egorov, E. Rabani, and B. J. Berne, J. Chem. Phys. 108, 1407 (1998).

${ }^{19}$ Q. Shi and E. Geva, J. Chem. Phys. 118, 8173 (2003).

${ }^{20}$ Q. Shi and E. Geva, J. Phys. Chem. A 107, 9059 (2003).

${ }^{21}$ Q. Shi and E. Geva, J. Phys. Chem. A 107, 9070 (2003).

${ }^{22}$ J. A. Poulsen, G. Nyman, and P. J. Rossky, J. Chem. Phys. 119, 12179 (2003).

${ }^{23}$ Q. Shi and E. Geva, J. Chem. Phys. 120, 10647 (2004).

${ }^{24}$ Q. Shi and E. Geva, J. Phys. Chem. A 108, 6109 (2004)

${ }^{25}$ S. A. Egorov, E. Rabani, and B. J. Berne, J. Chem. Phys. 110, 5238 (1999).

${ }^{26}$ H. Wang, X. Sun, and W. H. Miller, J. Chem. Phys. 108, 9726 (1998).

${ }^{27}$ W. H. Miller, J. Chem. Phys. 53, 3578 (1970).

${ }^{28}$ E. J. Heller, J. Chem. Phys. 94, 2723 (1981).

${ }^{29}$ K. G. Kay, J. Chem. Phys. 100, 4377 (1994)

${ }^{30}$ M. F. Herman and E. Kluk, Chem. Phys. 91, 27 (1984).

${ }^{31}$ E. Kluk, M. F. Herman, and H. L. Davis, J. Chem. Phys. 84, 326 (1986).

${ }^{32}$ D. Provost and P. Brumer, Phys. Rev. Lett. 74, 250 (1995).

${ }^{33}$ A. R. Walton and D. E. Manolopoalos, Mol. Phys. 87, 961 (1996).

${ }^{34}$ M. Ovchinnikov and V. A. Apkarian, J. Chem. Phys. 105, 10312 (1996).

${ }^{35}$ M. L. Brewer, J. S. Hulme, and D. E. Manolopoulos, J. Chem. Phys. 106, 4832 (1997).

${ }^{36}$ M. A. Sepúlveda and S. Mukamel, J. Chem. Phys. 102, 9327 (1995).

${ }^{37}$ M. A. Sepúlveda and S. Mukamel, Adv. Chem. Phys. 96, 191 (1996).

${ }^{38}$ C. F. Spencer and R. F. Loring, J. Chem. Phys. 105, 6596 (1996).

${ }^{39}$ S. A. Pentidis and R. F. Loring, Chem. Phys. Lett. 287, 217 (1998).

${ }^{40}$ M. Ovchinnikov and V. A. Apkarian, J. Chem. Phys. 108, 2277 (1998).

${ }^{41}$ M. Thoss and W. H. Miller, J. Chem. Phys. 112, 10282 (2000).

${ }^{42}$ H. Wang, M. Thoss, K. Sorge, R. Gelabert, X. Gimenez, and W. H. Miller, J. Chem. Phys. 114, 2562 (2001).

${ }^{43}$ X. Sun and W. H. Miller, J. Chem. Phys. 106, 916 (1997).

${ }^{44}$ M. Ovchinnikov and V. A. Apkarian, J. Chem. Phys. 106, 5775 (1997).

${ }^{45}$ N. Makri and K. Thompson, Chem. Phys. Lett. 291, 101 (1998).

${ }^{46}$ K. Thompson and N. Makri, Phys. Rev. E 59, R4729 (1999).

${ }^{47}$ K. Thompson and N. Makri, J. Chem. Phys. 13, 1343 (1999).

${ }^{48}$ W. H. Miller, Faraday Discuss. 110, 1 (1998).

${ }^{49}$ V. S. Batista, M. T. Zanni, B. Jefferys Greenblatt, D. N. Neumark, and W. H. Miller, J. Chem. Phys. 110, 3736 (1999).

${ }^{50}$ E. Rabani, S. A. Egorov, and B. J. Berne, J. Chem. Phys. 109, 6376 (1998).

${ }^{51}$ J. R. Klauder and P. W. Anderson, Phys. Rev. 125, 912 (1962).

${ }^{52}$ M. Berg, C. A. Walsh, L. R. Narasimhan, K. A. Litttau, and M. D. Fayer, J. Chem. Phys. 88, 1564 (1988).

${ }^{53}$ Y. S. Bai and M. D. Fayer, Phys. Rev. B 39, 11066 (1989).

${ }^{54}$ A. Suárez and R. Silbey, Chem. Phys. Lett. 218, 445 (1994). 
${ }^{55}$ E. Geva and J. L. Skinner, J. Chem. Phys. 107, 7630 (1997).

${ }^{56}$ E. Rabani, S. A. Egorov, and B. J. Berne, J. Chem. Phys. 103, 9539 (1999).

${ }^{57}$ T. Kalbfleisch, R. Fan, J. Roebber, P. Moore, E. Jacobsen, and L. D. Ziegler, J. Chem. Phys. 103, 7673 (1995).

${ }^{58}$ L. E. Fried and S. Mukamel, Adv. Chem. Phys. 84, 435 (1993).
${ }^{59}$ J. A. Barker, D. Henderson, and F. F. Abraham, Physica A 106, 226 (1981).

${ }^{60}$ M. P. Allen and D. J. Tildesley, Computer Simulation of Liquids (Clarendon, Oxford, 1987).

${ }^{61}$ S. Jang and G. A. Voth, J. Chem. Phys. 107, 9514 (1997). 